\title{
Study of The Effect of Adding a Blend of Engineering Polymers on the Rheological Modifications of Sulfurated Asphalt
}

\author{
Mahmoud Fadel Abass ${ }^{1 *}$, Ammar Ahmed Hamdoon² \\ 1*Ministry of Education/ Nineveh, Education Directorate, Mosul, Iraq \\ ${ }^{2}$ Department of Chemistry, College of Education for Pure Science, University of Mosul, Mosul, Iraq \\ E-mail: ${ }^{1 *}$ Mahmoud.esp106@ student.uomosul.edu.iq, ${ }^{2}$ ammarhamdoon@ uomosul.edu.iq
}

(Received April 15, 2021; Accepted June 23, 2021; Available online September 01, 2021)

DOI: 10.33899/edusj.2021.129947.1157, (C) 2021, College of Education for Pure Science, University of Mosul.

This is an open access article under the CC BY 4.0 license (http://creativecommons.org/licenses/by/4.0/)

\begin{abstract}
In this study, a mixture of two polymers consisting of commercial adhesive [ethylene vinyl acetate (EVA)] and melamine (M) Which were used in this study.

Different weights of both polymers were used with total weights equal for $(1 \mathrm{~g})$. The asphalt was treated with the above mixture in the presence of $1 \%$ by weight of sulfur at $\left(180^{\circ} \mathrm{C}\right)$ for one hour.

The rheological properties of the modified samples and original one were measured, which included measuring the ductility, penetration, and softining point as well as calculating the penetration index and percentage of the separated asphaltens .

After that, the best samples obtained from the above modification process were taken, and several interactions were performed on it in order to determine each of :

The optimum conditions for the conversion process have been established and include the percentage of added sulfur and the temperature the best time, the best weights of the mixture, and the best addition sequence.

After that, the best sample obtained from this study was taken and the marshals test with chemical immersion was performed, as well as the effect of aging on this sample was studied.

The study gave good results in the field of asphalt resistance to the deformation processes which were carried out on it.
\end{abstract}

Keyword: Polymeric mixtures , Rheological Properties , Asphalt, Sulfur

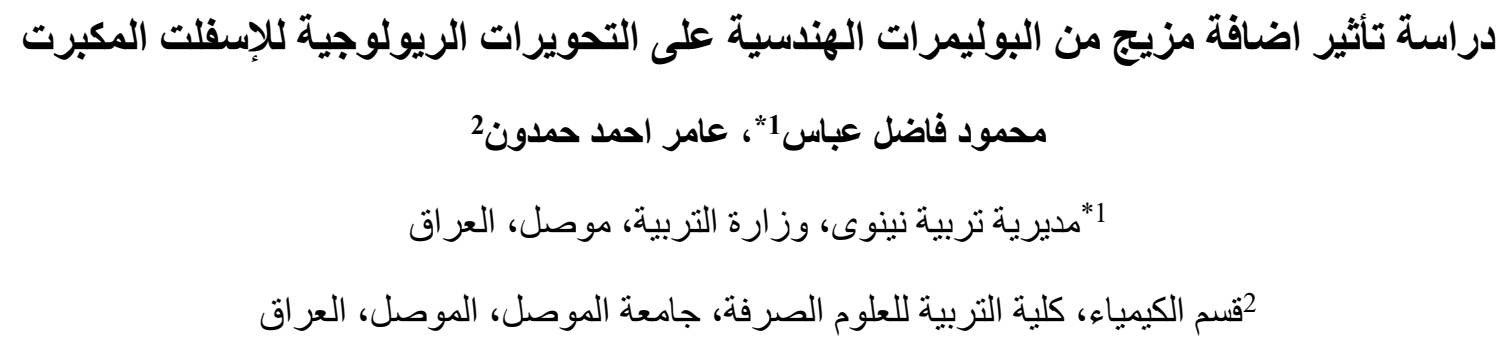


استخدم في هذه الدر اسة مزيج مكون من بوليمرين مصنعين هما اللاصنق التجاري المُكون بصورة رئيسة من الاثيلين فينايل استيت

(EVA)

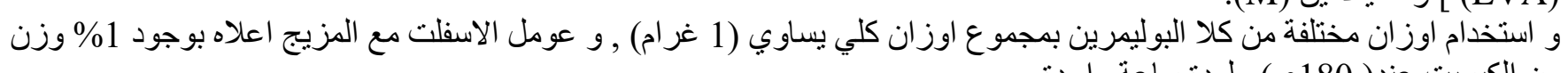

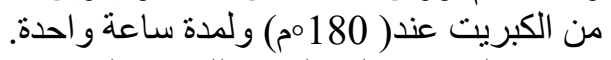

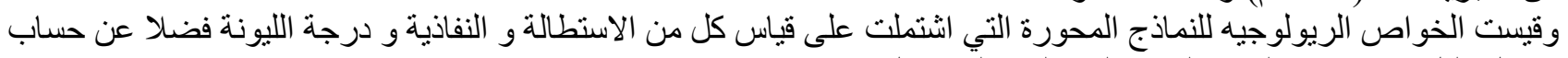

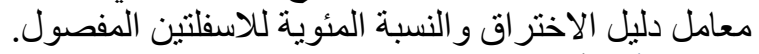

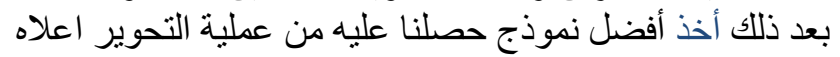

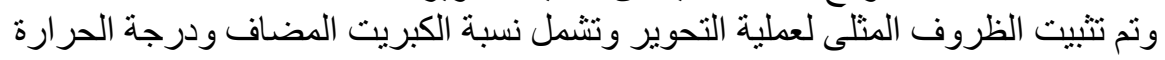

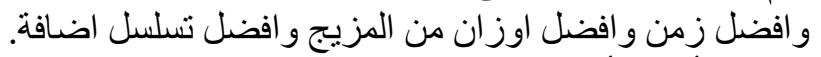
بعد ذلك أخذت أفضل نموذج نم ارذان الحصول النزيج عليه من هذه الدر اسة و اجري عليه فحص المارشال و الغمر الكيميائي فضلا عن در اسة اثر

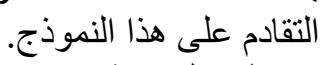
واعطت الدر اسة نتائج جيدة في مجال مقاومة الإسفلت لعمليات التشويه التي أجريت عليه. الكلمات المفتاحية : المزائج البوليمرية , الخواص الريولوجية , إسفلت ,كبريت

\section{المقدمة :}

يعرف الإسفلت على انه مادة سائلة ثقيلة او شبه صلبة ذات لزوجة عالية ويتم الحصول عليه من عملية التقطير المباشر للنفط الخام(2,1).

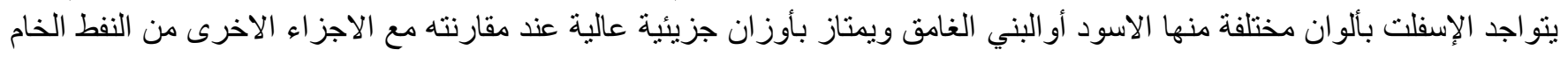

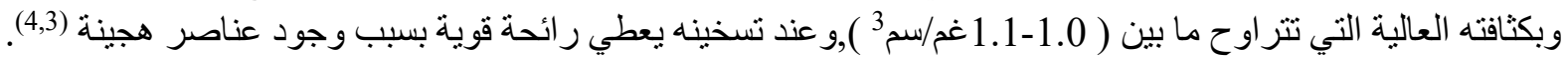

كما يمكن إعطاء الإسفلت تعريف آخر بأنه تللك المادة البتيومينية الثقيلة التي تبقى بعد ازالة المشتقات النفطية الخفيفة القابلة للنطاير و التقطير (5).

يتكون الإسفلت بصورة رئيسة من مركبات هايدروكاربونات (بارفينية ونفتينية و اروماتية ) كما يحتوي على العديد من المركبات الحلقية

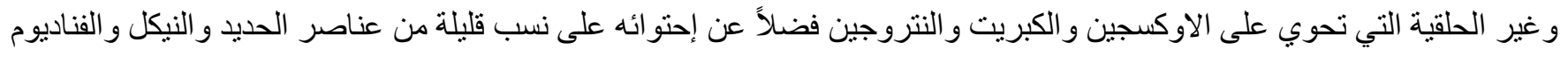

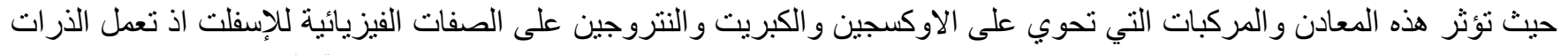

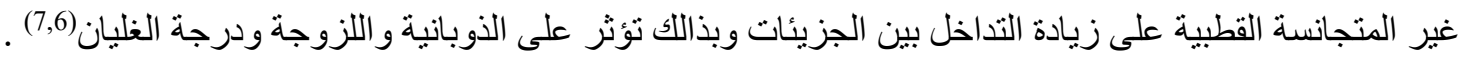
يمناز الإسفلت بخموله الكيميائي والمتوافق مع خواصده الفيزيائية وهو الأمر الذي يجعل منه مؤهل لاستعمالات واسعة في البناء

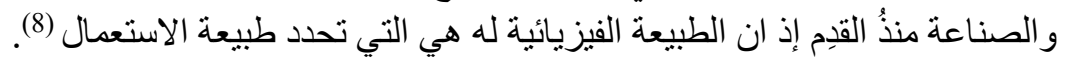

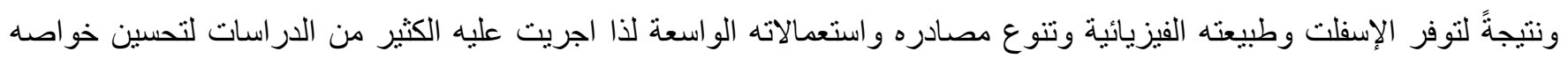

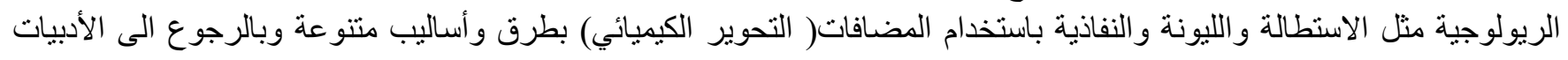
نرى العديد من الدر اسات في هذا المجال ومنها:-

ما قام باه(Cunha) (9) وجماعته بدر اسة الخو اص الريولوجية للإسفلت المحور بالألياف الطبيعية ومضادات الأكسدة حيث تؤدي اضافة

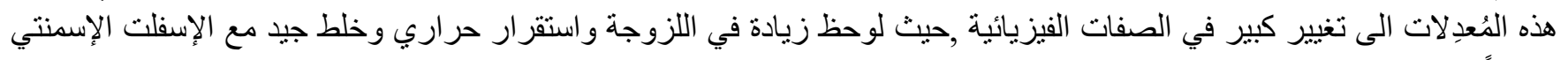

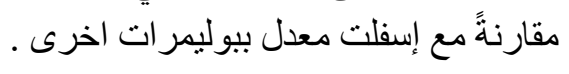

وتمكنت ( Adil)(10) من استخدام بوليمر ات مستهلكة مثل البولي ( اثيلين - تيرفثاليت) المأخوذة من قناني المياه البلاستيكية في تحوير

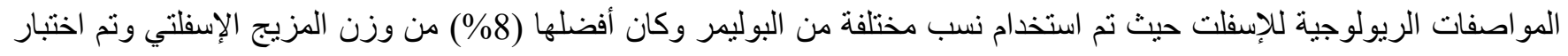

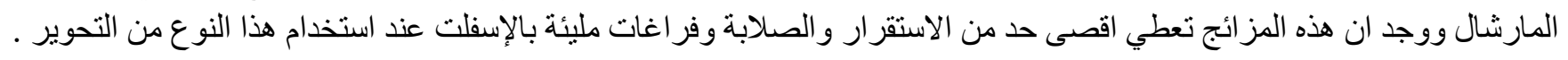


ودرس كل من (Gau \& Wu) (11) امكانية إضافة نوع خاص من الالياف (Basalt) الى ص صفوف الخرسانة الاسفلتية وقد تم قياس

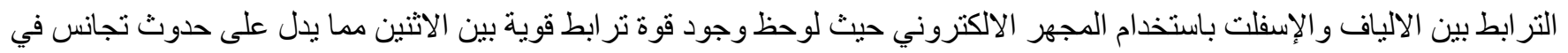

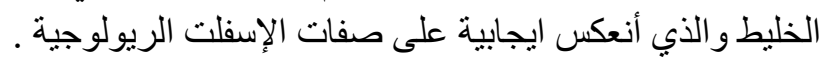

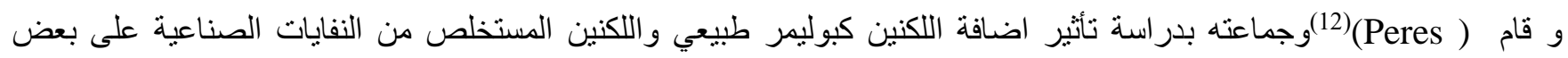

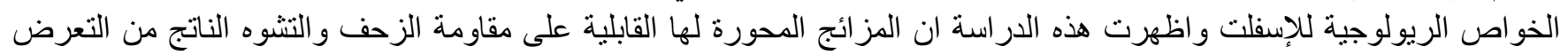
للحر ارة العالية.

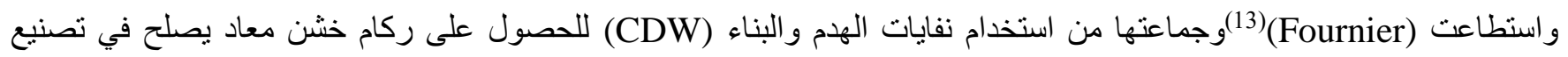

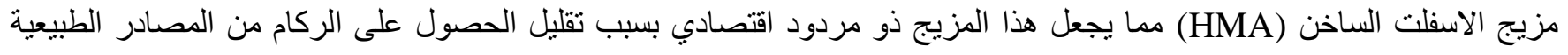
وكذللك المحافظة على بيئة صحية ونظيفة.

ودرس (Ahmed \& Hamdoon) استخدام اللكنين في التحوير الريولوجي لإسفلت الدورة وبمسارات مختلفين الاول باستخدام

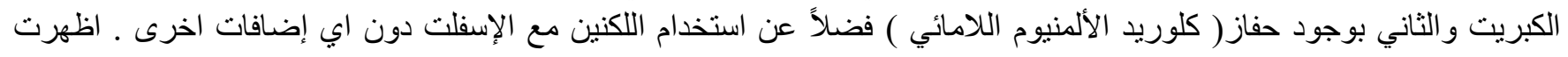
الدر اسة انتاج نماذج إسفلتية ذات مو اصفات مختلفة عن الإسفلت الاصل يمل يمكن استعمالها في مجال التبليط.

فضلاً عن ذلك فقد تمكن الباحثان (Ahmed \& Hamdoon) من دراسة الخواص الريولوجية للإسفلت المحور باللاصف التجاري

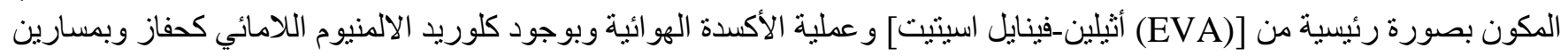

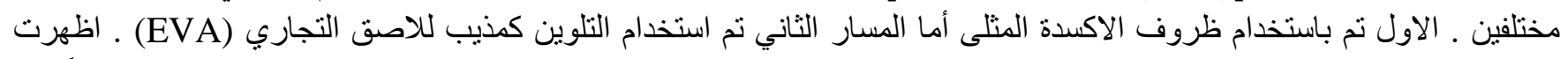

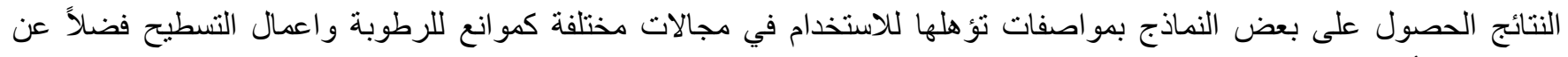
الاستعمال الأهم الاوهو التبليط.

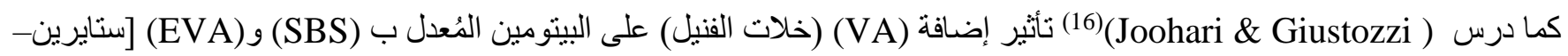

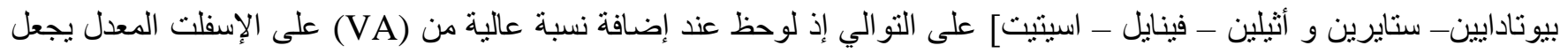

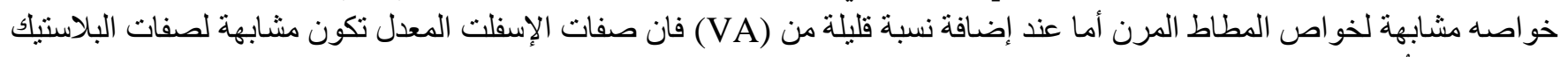
الصلب إذ خلصصت الدر اسة ومن خلال نتائج التحليل الحراري اند الزيادة من (VA) تعيق تبلور البوليمر داخل البيتومين .

وقام كل من ( Mashaan \& Nikraz) (17) بدر اسة الخو اص الهندسية للمادة الر ابطة الإسفلتية المعدلة بالنفايات المحلية من مادة البولي

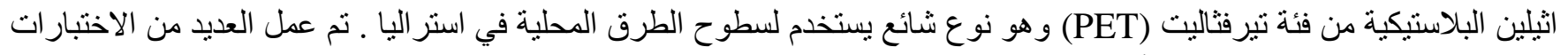

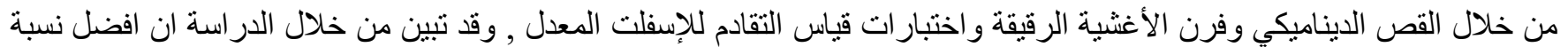

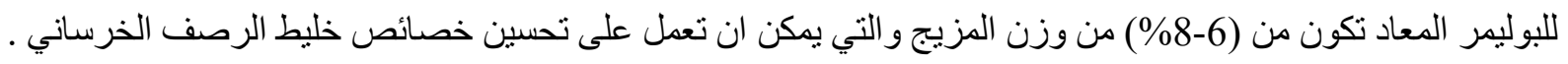

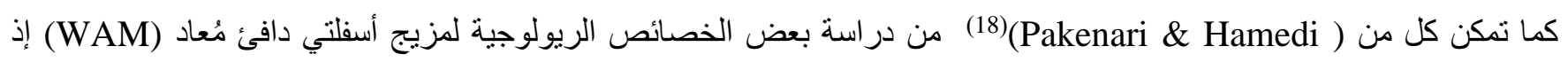

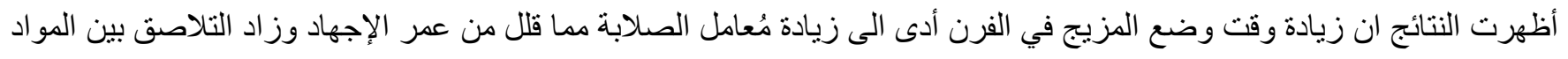

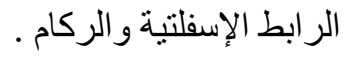

ودرس (Hamedi \& Joubani) تأثير الإسفلت المُعدل بالبوليمر (SBR) مطاط ستايرين - بيوتادايين على تحسين قابلية الرطوبة

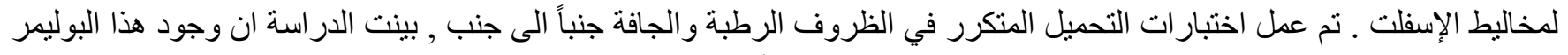

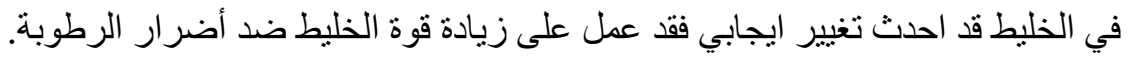

ان عملية الحصول على نماذج أسفلتيه جيدة بحيث يمكن ان تستخدم لأغر اض مختلفة ليست بالعملية السهلة سيما ان استخدام الأسفلت

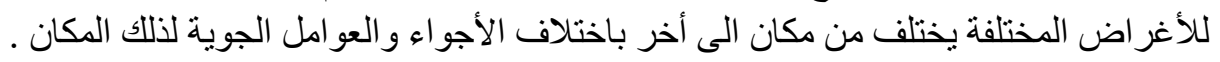




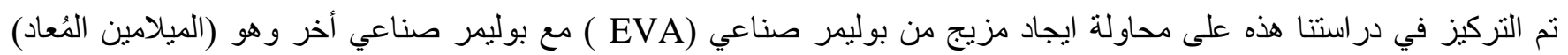

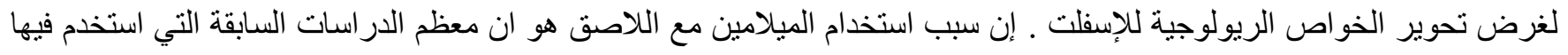

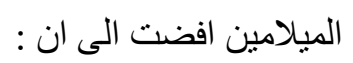

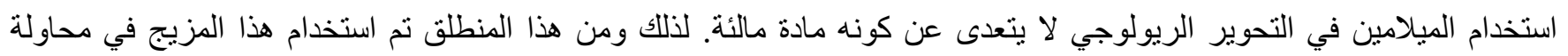

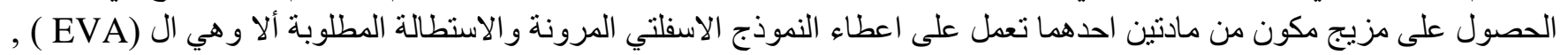

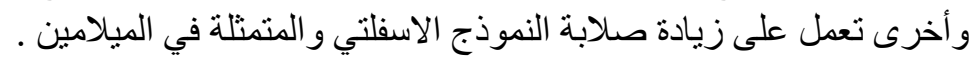

$$
\begin{aligned}
& \text { الجزء العملي } \\
& \text { اولاً : المواد المستخدمة }
\end{aligned}
$$

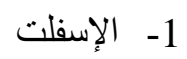

$$
\begin{aligned}
& \text { تم الحصول عليه من وزارة النفط العر اقية (مصافي شركة نفط الثمال) }
\end{aligned}
$$

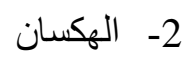

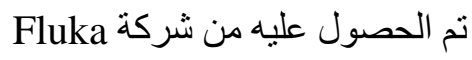

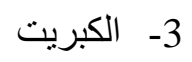

$$
\begin{aligned}
& \text { تم الحصول عليه من شركة Judex Chernicals البريطانية } \\
& \text { 4- لاصق تجاري جُل تركيبه من الأثيلين- فينايل اسيتيت ( EVA) (20) } \\
& \text { تم الحصول عليها من الاسو اق المحلية }
\end{aligned}
$$

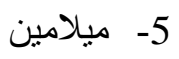

$$
\begin{aligned}
& \text { تم الحصول عليها من بقايا المو اد المصنعة منها } \\
& \text { ثانياً : الأجزةة المستخدمة }
\end{aligned}
$$

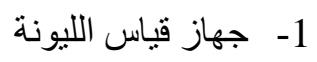

$$
\begin{aligned}
& \text { 2- - جهاز قياس الاستطالة وهو من نوع (YUFENG) ذو من منشأ صيني }
\end{aligned}
$$

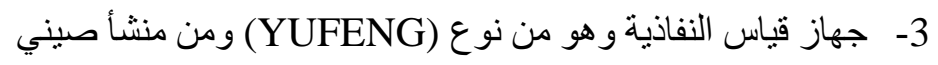

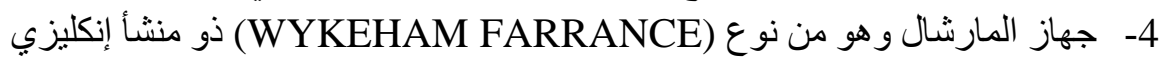

$$
\begin{aligned}
& \text { 5- فرن التقادم وهو من نوع من نوع MENC1321 من منشأ إنكليزي } \\
& \text { ثالثاً : الطرائق العملية } \\
& \text { 1- - - المعاملة الحر ارية للميلامين }
\end{aligned}
$$


عوملت بقايا الميلامين عند مدى حراري يتز اوح بين (50-300×م) وبزمن ساعة واحدة عند كل درجة حرارية وبزيادة (50مم) لكل قراءة. 2- 2 التحوير الريولوجي للإسفلت عومل الإسفلت مع مزيج من (EVA : ميلامين) بحيث يكون الوزن النهائي للمزيج (1غم) بوجود 1\% وزناً من الكبريت لمدة

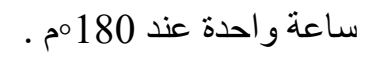
3- قياس الخو اص الريولوجية

نم قياس الخواص الريولوجية لكل من الإسفلت الاصل والنماذج الناتجة من الخطوة (2) و التي شمل قياس كل من الاسنطالة(21)

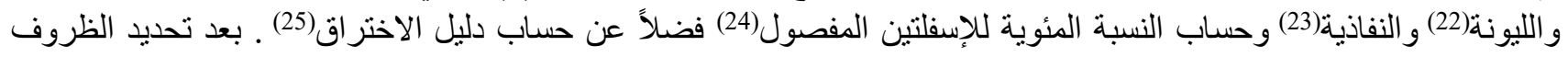

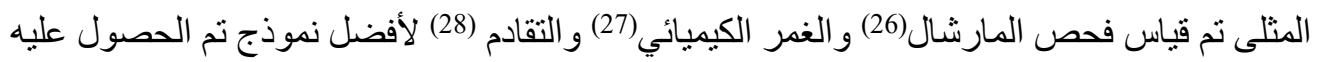

$$
\text { 4- ت تحديد الظروف المُنلى للتحوير الريولوجي }
$$

أخذ أفضل النماذج التي تم الحصول عليها من الخطوة (2) و أجريت عليها تفاعلات عدة لتحديد كل من:

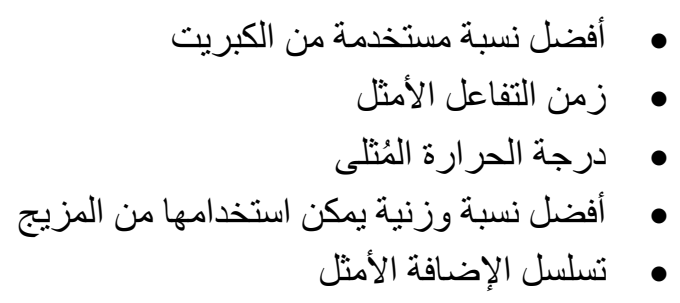

النتائج والمناقشة :

يعد امر الحصول على إسفلت ذا خو اص ريولوجية ممتازة تتلائم مع الغايات التي تستخدم من اجلها أمر بالغ الأهمية ـ من در استنا هذه نم

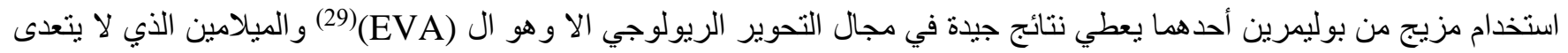

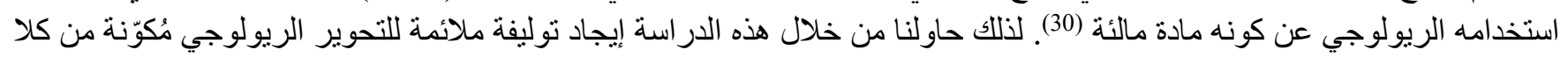
البوليمرين .

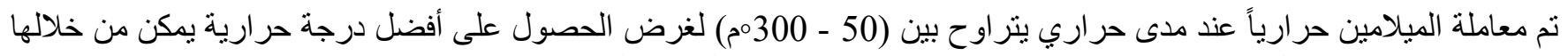

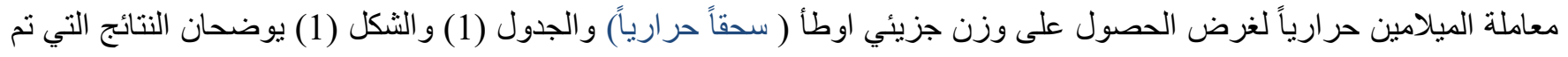

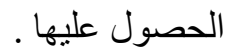

الجدول (1) يوضح نتائج المعاملة الحرارية للميلامين

\begin{tabular}{|c|c|}
\hline (الوزن & 1 \\
\hline 0 & 0.99 \\
\hline 50 & 0.916 \\
\hline 100 & \\
\hline
\end{tabular}




\begin{tabular}{|c|c|}
\hline 150 & 0.911 \\
\hline 200 & 0.892 \\
\hline 250 & 0.625 \\
\hline 300 & 0.448 \\
\hline
\end{tabular}

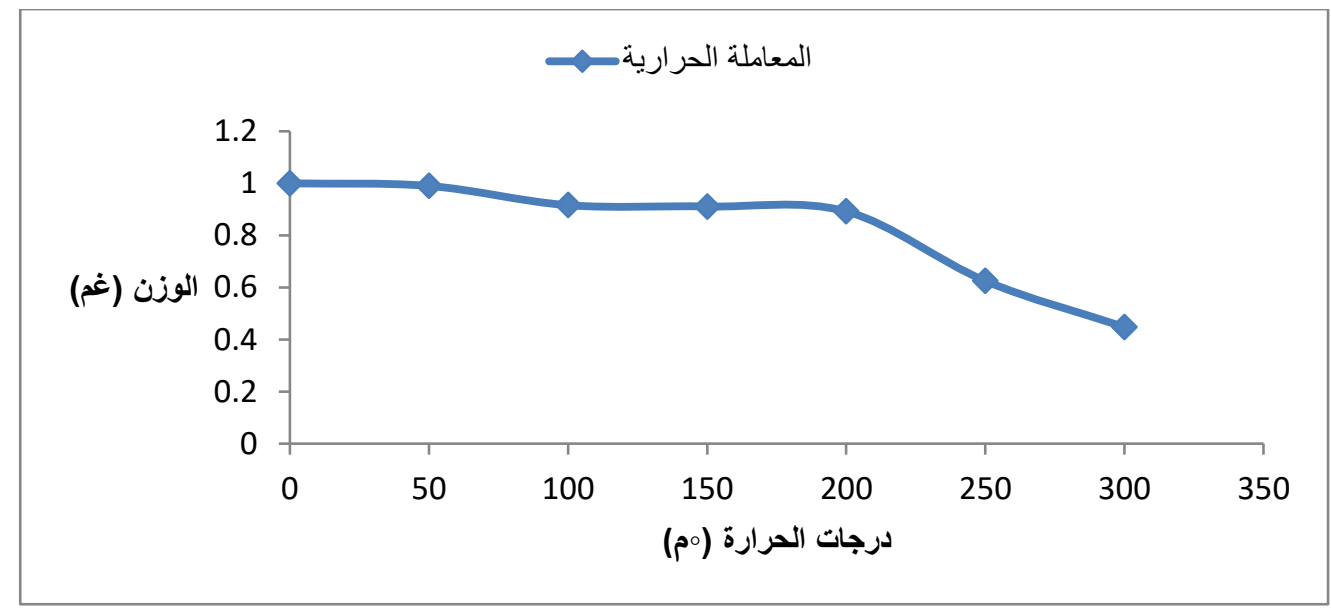

الثكل (1) المعاملة الحرارية للميلامين

يتضح من الثكل (1) والجدول (1) أن أفضل درجة حرارية لمعاملة الميلامين كانت (200)م (ليتم بعد ذلك طحنه وتحويله الى مسحوق

عومل إسفلت الأصل مع مزيج كلا البوليمرين بأوزان مختلفة عند 180مم وبوجود 1\% وزناً كبريت ولمدة ساعة واحدة والجدول (2)

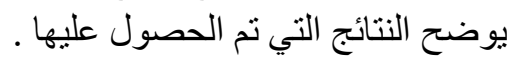

الجدول (2) : الخواص الريولوجية للإسفلت المعامل مع مزائج مختلفة من (M, EVA ) بوجود 1\% وزناً كبريت عند 180مم لمدة

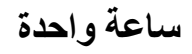




\begin{tabular}{|c|c|c|c|c|c|c|c|}
\hline \multirow{2}{*}{ الإسفتلتين } & \multirow{2}{*}{ الاختراق(PI) دليل } & \multirow{2}{*}{ 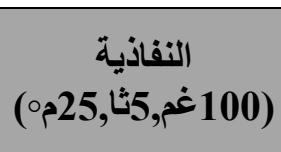 } & \multirow{2}{*}{ الليونة } & \multirow{2}{*}{$\begin{array}{c}\text { الاستطالة } \\
\text { ( 25,cm) }\end{array}$} & \multicolumn{2}{|c|}{ المُضافات } & \multirow{2}{*}{ الانموذج } \\
\hline & & & & & EVA | & M & \\
\hline 16.8 & -1.349 & 46.7 & 50 & $150<$ & 0 & 0 & $\mathrm{AS}_{0}$ \\
\hline 24 & -0.718 & 42.73 & 53.5 & $150<$ & 0.1 & 0.9 & $\mathrm{AS}_{1}$ \\
\hline 23.2 & -0.387 & 42.75 & 55 & $150<$ & 0.2 & 0.8 & $\mathrm{AS}_{2}$ \\
\hline 23.5 & -1.060 & 42.71 & 53 & $150<$ & 0.3 & 0.7 & $\mathrm{AS}_{3}$ \\
\hline 23.4 & -0.612 & 42.70 & 54 & $150<$ & 0.4 & 0.6 & $\mathrm{AS}_{4}$ \\
\hline 23.8 & -0.555 & 42.85 & 54.2 & $150<$ & 0.5 & 0.5 & $\mathrm{AS}_{5}$ \\
\hline 22.9 & -0.726 & 42.98 & 53.4 & $150<$ & 0.6 & 0.4 & $\mathrm{AS}_{6}$ \\
\hline 22.1 & -0.927 & 42.11 & 52.5 & $150<$ & 0.7 & 0.3 & $\mathrm{AS}_{7}$ \\
\hline 21.9 & -1.766 & 43.09 & 49 & $150<$ & 0.8 & 0.2 & $\mathrm{AS}_{8}$ \\
\hline 21.3 & -1.2 & 43.55 & 48 & $150<$ & 0.9 & 0.1 & $\mathrm{AS}_{9}$ \\
\hline
\end{tabular}

: AS : الإسفلت الاصل دون اي مُضافات
: M EVA

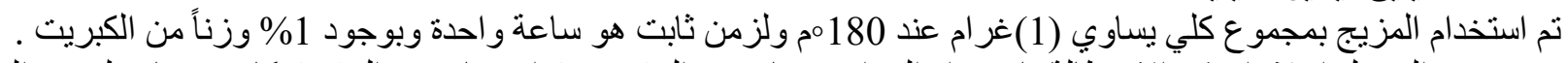

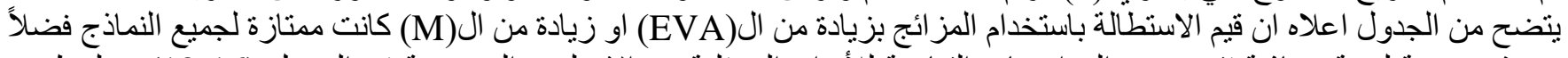

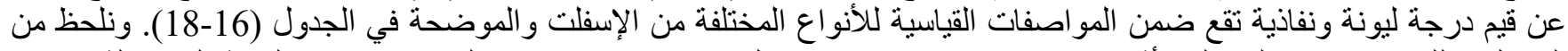

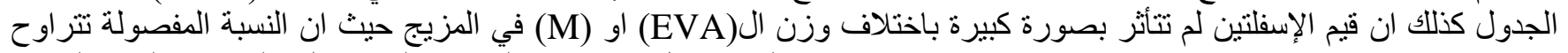

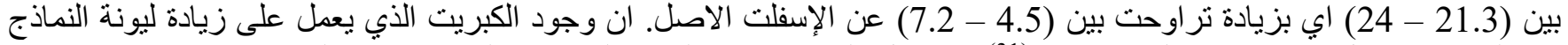

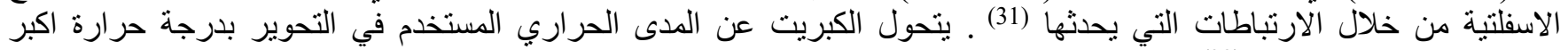

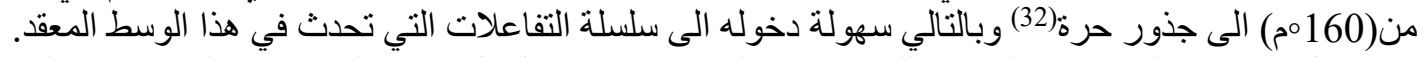
يعطي ال(EVA) المزيد من المرونة للنماذج الإسفلتية في حين يعمل ال(M) على اعطاء النماذج الإسفلتية الصلابة المطلوبة بوصفه

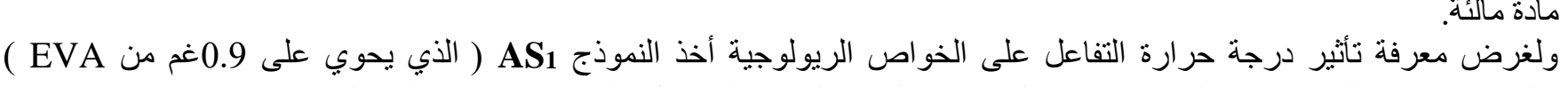

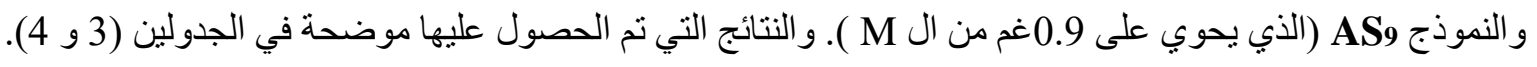

الجدول (3) : الخواص الريولوجية للنموذج (AS1) عند درجات حرارية مختلفة بوجود 1\% وزناً من الكبريت وزمن ساعة واحدة

\begin{tabular}{|c|c|c|c|c|c|c|}
\hline الإسفلتين & الاختراق(PI) دليل & 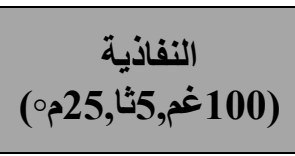 & الليوجة & $\begin{array}{c}\text { (الاستطالة } \\
\text { (ال25,cm) }\end{array}$ & الحرارة & الأنموذج \\
\hline 16.8 & -1.349 & 46.7 & 50 & $150<$ & 0 & $\mathrm{AS}_{0}$ \\
\hline 29.4 & -0.059 & 41.65 & 56.8 & 125 & 120 & $\mathrm{AS}_{1}$ \\
\hline 25.3 & -1.001 & 42.88 & 52.5 & $150<$ & 150 & $\mathrm{AS}_{1}$ \\
\hline 24 & -0.718 & 42.33 & 53.5 & $150<$ & 180 & $\mathrm{AS}_{1}$ \\
\hline 32 & -0.6 & 41.98 & 54.2 & 113 & 200 & $\mathrm{AS}_{1}$ \\
\hline
\end{tabular}

الجدول (4) : الخواص الريولوجية للنموذج (AS9) عند درجات حرارية مختلفة بوجود 1\% وزناً من الكبريت وزمن ساعة واحدة 


\begin{tabular}{|c|c|c|c|c|c|c|}
\hline الإسفلتين & الاختراق(PI دليل & 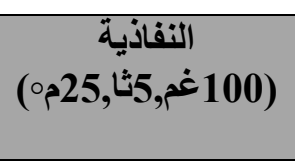 & الليونة & $\begin{array}{c}\text { الاستطالة } \\
\text { (25,cm)مه }\end{array}$ & الحرارة & الأنموذج \\
\hline 16.8 & -1.349 & 46.7 & 50 & $150<$ & 0 & $\mathrm{AS}_{0}$ \\
\hline 29.5 & -0.719 & 42.71 & 53.5 & $150<$ & 120 & $\mathrm{AS}_{9}$ \\
\hline 23 & -1.45 & 43.1 & 52 & $150<$ & 150 & $\mathrm{AS}_{9}$ \\
\hline 21.3 & -1.2 & 43.55 & 48 & $150<$ & 180 & $\mathrm{AS}_{9}$ \\
\hline 22.8 & -1.261 & 43.45 & 51 & $150<$ & 200 & $\mathrm{AS}_{9}$ \\
\hline
\end{tabular}

يتضح من الجدولين ان درجة الحرارة التي تم استخدامها كدرجة حرارة افتراضية للتفاعل كانت بالفعل درجة الحر ارة الئنلى التي من

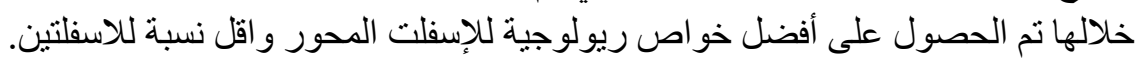

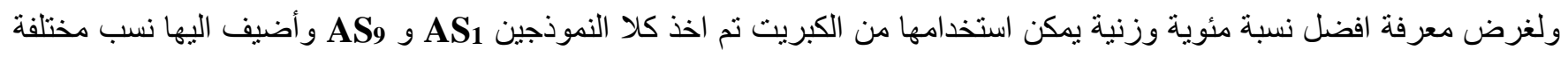

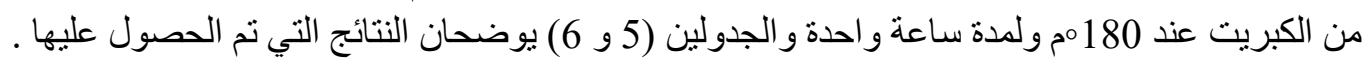
الجدول (5) : الخواص الريولوجية للنموذج AS1 باستخدام نسب وزنية مختلفة من الكبريت عند 180م وزمن ساعة واحدة

\begin{tabular}{|c|c|c|c|c|c|c|}
\hline الإسفلتين & الاختراق(PI) دليل & 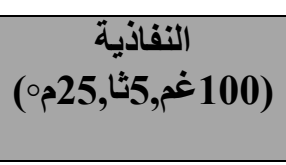 & الليونة & $\begin{array}{c}\text { الاستطالة } \\
\text { (م)25,cm) }\end{array}$ & $\begin{array}{c}\text { الكبريت } \\
\text { (\%بة) }\end{array}$ & الأنموذج \\
\hline 16.8 & -1.349 & 46.7 & 50 & $150<$ & 0 & $\mathrm{AS}_{0}$ \\
\hline 31 & -0.617 & 42.50 & 54 & 110 & 0.25 & $\mathrm{AS}_{1}$ \\
\hline 29.2 & -0.27 & 42.88 & 55.5 & 124 & 0.5 & $\mathrm{AS}_{1}$ \\
\hline 24 & -0.718 & 42.73 & 53.5 & $150<$ & 1 & $\mathrm{AS}_{1}$ \\
\hline 26.4 & -0.22 & 42.60 & 55.8 & 137 & 2 & $\mathrm{AS}_{1}$ \\
\hline
\end{tabular}

الجدول (6) : الخواص الريولوجية للنموذج AS9 باستخدام نسب وزنية مختلفة من الكبريت عند 180مم وزمن ساعة واحدة

\begin{tabular}{|c|c|c|c|c|c|c|}
\hline الإسفلتين & الاختراق(PI) دليل & 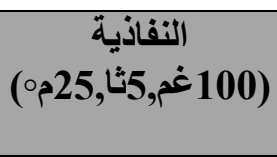 & الليونة & $\begin{array}{c}\text { الاسنطالة } \\
\text { (الم,25,cm) }\end{array}$ & $\begin{array}{l}\text { الكبريت } \\
\text { (\%بة } \\
\text { (\%) }\end{array}$ & الأنموذج \\
\hline 16.8 & -1.349 & 46.7 & 50 & $150<$ & 0 & $\mathrm{AS}_{0}$ \\
\hline 22.1 & -1.02 & 43.61 & 52 & $150<$ & 0.25 & $\mathrm{AS}_{22}$ \\
\hline 21.2 & -1.121 & 43.57 & 51.2 & $150<$ & 0.5 & $\mathrm{AS}_{23}$ \\
\hline 21.3 & -1.2 & 43.55 & 48 & $150<$ & 1 & $\mathrm{AS}_{24}$ \\
\hline 20.9 & -1.38 & 43.56 & 50.5 & $150<$ & 2 & $\mathrm{AS}_{25}$ \\
\hline
\end{tabular}

يتضح كنلك من كلا الجدولين ان نسبة 1\% وزناً من الكبريت كانت هي النسبة الأمثل لإجر اء أفضل تحوير ريولوجي. 
فضلاً عن ذلك حدد زمن التفاعل الأمثل لكلا النموذجين الذين تم اختبار هما بوصفِهما أفضل النماذج التي تم الحصول عليها ( AS

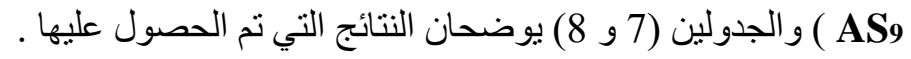
الجدول (7) : الخواص الريولوجية للنموذج AS1 عند أزمان مختلفة بوجود 1\% وزناً من الكبريت عند 180مم

\begin{tabular}{|c|c|c|c|c|c|c|}
\hline الإسفلتين & الاختراق(PI) دليل & 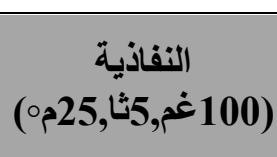 & الليونة & 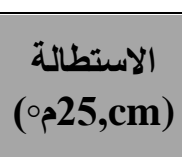 & (دقيقة) & الأنموذج \\
\hline 16.8 & -1.349 & 46.7 & 50 & $150<$ & 0 & $\mathrm{AS}_{0}$ \\
\hline 22.9 & -1.4 & 43.09 & 50.5 & $150<$ & 30 & $\mathrm{AS}_{1}$ \\
\hline 24 & -0.718 & 42.73 & 53.5 & $150<$ & 60 & $\mathrm{AS}_{1}$ \\
\hline 28.3 & -0.425 & 41.98 & 55 & 136.5 & 90 & $\mathrm{AS}_{1}$ \\
\hline 30.2 & +0.08 & 41.54 & 57.5 & 118.3 & 120 & $\mathrm{AS}_{1}$ \\
\hline
\end{tabular}

الجدول (8) : الخواص الريولوجية للنموذج 9S عند أزمان مختلفة بوجود 1\% وزناً من الكبريت عند 180مم

\begin{tabular}{|c|c|c|c|c|c|c|}
\hline الإسفلتين & الاختراق(PI) دليل & 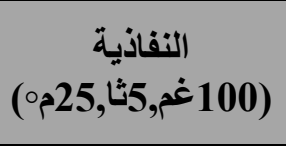 & الليونة & $\begin{array}{c}\text { الاستطالة } \\
\text { (المث25,cm) }\end{array}$ & (لالزمة) & الأنموذج \\
\hline 16.8 & -1.349 & 46.7 & 50 & $150<$ & 0 & $\mathrm{AS}_{0}$ \\
\hline 20.8 & -1.852 & 44 & 48.5 & $150<$ & 30 & $\mathrm{AS}_{9}$ \\
\hline 21.3 & -1.2 & 43.55 & 48 & $150<$ & 60 & $\mathrm{AS}_{9}$ \\
\hline 22.7 & -1.521 & 43.32 & 50 & $150<$ & 90 & $\mathrm{AS}_{9}$ \\
\hline 23 & -1.471 & 43.12 & 50.2 & 148 & 120 & $\mathrm{AS}_{9}$ \\
\hline
\end{tabular}

يتضح من الجدولين ان الزمن الذي تم اختياره في بداية التفاعل (60 دقيقة) كان بالفعل الزمن الأمثل لإجراء التحويرات الريولوجية .

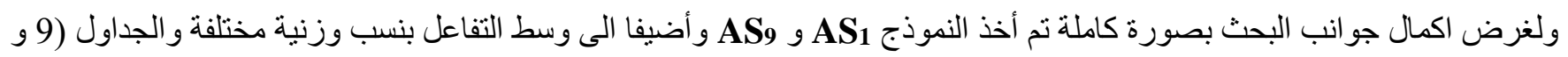

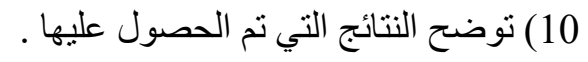

الجدول (9) : الخواص الريولوجية للإسفلت المعامل مع نسب مختلفة من مزيج (M: EVA) (0.9:0.1) بوجود 1\% وزناً من

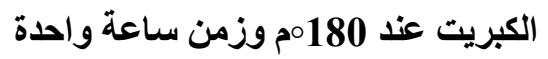

\begin{tabular}{|c|c|c|c|c|c|c|}
\hline الإسفلتين & الاختراق(PI) دليل & 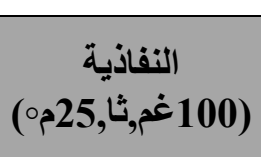 & الليونة & $\begin{array}{c}\text { الاستطالة } \\
\text { (ال25,cm) }\end{array}$ & المئوية & الأنموذج \\
\hline 16.8 & -1.349 & 46.7 & 50 & $150<$ & 0 & $\mathrm{AS}_{0}$ \\
\hline 30 & -0.382 & 41.98 & 55.2 & 118.5 & 0.5 & $\mathrm{AS}_{10}$ \\
\hline 26.5 & -0.625 & 42.35 & 54 & 124 & 0.75 & $\mathrm{AS}_{11}$ \\
\hline 24 & -0.718 & 42.73 & 53.5 & $150<$ & 1 & $\mathrm{AS}_{12}$ \\
\hline 22.5 & -1.75 & 43.49 & 49 & $150<$ & 2 & $\mathrm{AS}_{13}$ \\
\hline 31.2 & -0.427 & 41.94 & 55 & 117 & 3 & $\mathrm{AS}_{14}$ \\
\hline
\end{tabular}


الجدول (10) : الخواص الريولوجية للإسفلت المعامل مع نسب مختلفة من مزيج (M: EVA) (0.1:0.9) بوجود \% 1زوناً من الكبريت عند 180مم وزمن ساعة واحدة الخدارة

\begin{tabular}{|c|c|c|c|c|c|c|}
\hline الإسفلتين & الاختراق(PI دليل & 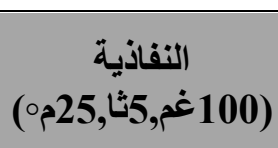 & الليونة & $\begin{array}{c}\text { الاستطالة) } \\
\text { (25,cm)مهo }\end{array}$ & اللوزئوية & الأنموذج \\
\hline 16.8 & -1.349 & 46.7 & 50 & $150<$ & 0 & $\mathrm{AS}_{0}$ \\
\hline 31 & -0.475 & 41.88 & 54.8 & 117.2 & 0.5 & $\mathrm{AS}_{15}$ \\
\hline 20.9 & -1.793 & 43.59 & 47.8 & $150<$ & 0.75 & $\mathrm{AS}_{16}$ \\
\hline 21.3 & -1.2 & 43.55 & 48 & $150<$ & 1 & $\mathrm{AS}_{17}$ \\
\hline 23.1 & -1.75 & 43.52 & 49 & $150<$ & 2 & $\mathrm{AS}_{18}$ \\
\hline 25.8 & -0.64 & 42.98 & 53.8 & 145 & 3 & $\mathrm{AS}_{19}$ \\
\hline
\end{tabular}

يتضح من كلا الجدولين ان استخدام هذا المزيج وبأي نسبة وزنية اعطى خواص ريولوجية ممنازة ومطابقة للنماذج القياسية المُوضحة

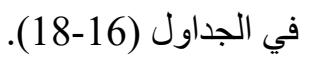

ولغرض اكمال ضبط جميع الظروف الخاصة بتفاعل التحوير الريولوجي تم تحديد افضل مسار للإضافة بالإمكان استخدامه للحصول

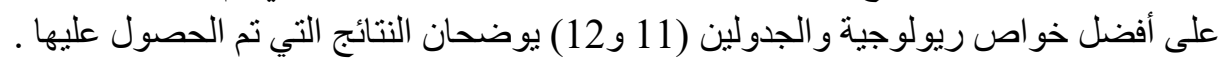

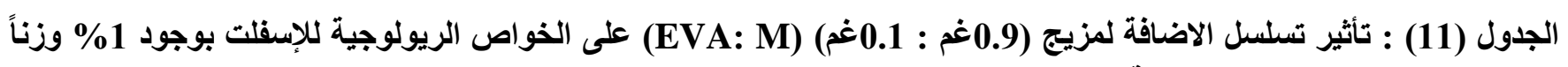

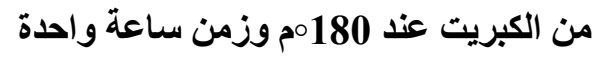

\begin{tabular}{|c|c|c|c|c|c|c|}
\hline الإسفلتين & الاختراق(PI) دليل & 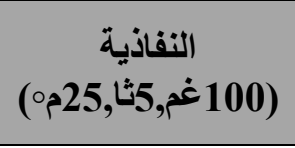 & الليوجة & $\begin{array}{c}\text { الاستطالة) } \\
\text { (م)25,cm) }\end{array}$ & تاضلسلة & الأنموذج \\
\hline 16.8 & -1.349 & 46.7 & 50 & $150<$ & 0 & $\mathrm{AS}_{0}$ \\
\hline 24 & -0.718 & 42.73 & 53.5 & $150<$ & اول المضافيع & $\mathrm{AS}_{20}$ \\
\hline 29.2 & -0.625 & 42.32 & 54 & 127 & 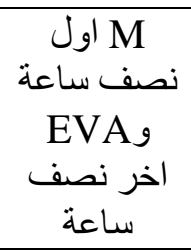 & $\mathrm{AS}_{21}$ \\
\hline 32 & +0.745 & 41.05 & 61 & 80 & نصف ساعة اخد & $\mathrm{AS}_{22}$ \\
\hline
\end{tabular}

AS0

الجدول (12) : يوضح تسلسل الاضافة لمزيج (18.1غم : 0.9 غم) (EVA: M) على الخواص الريولوجية للإسفلت بوجود 1\% وزناً من الكبريت عند 180مم وزمن قدره ساعة واحدة 


\begin{tabular}{|c|c|c|c|c|c|c|}
\hline ا الإسفاتثين & الاختراق(PI) دليل & 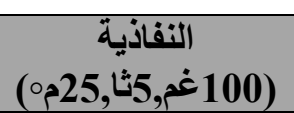 & الليونة (مه) & $\begin{array}{c}\text { الاستطالة) } \\
\text { (0م25,cm) }\end{array}$ & الاضلسلة & الأنموذج \\
\hline 16.8 & -1.349 & 46.7 & 50 & $150<$ & 0 & $\mathrm{AS}_{0}$ \\
\hline 21.3 & -1.2 & 43.55 & 48 & $150<$ & المضافيع & $\mathrm{AS}_{23}$ \\
\hline 23.2 & -1.213 & 43.46 & 51.2 & $150<$ & نصف ساعة & $\mathrm{AS}_{24}$ \\
\hline 22.1 & -1.761 & 43.52 & 49 & $150<$ & نصف ست ساعة اخرة & $\mathrm{AS}_{25}$ \\
\hline
\end{tabular}

الخو اص الريولوجية , اما الجدول (12) يتضح من الجدول (11) ان استخدام الميلامين في بداية التفاعل كان الافضل من ناحية تحسين

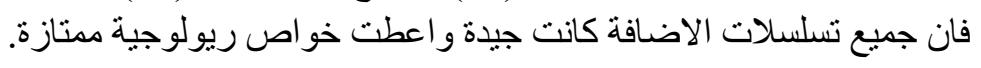

ولغرض معرفة امكانية استخدام النموذج المحور في عملية التبليط تم أخذ النموذج (AS5 ) وأجري عليه اختبار المارشال والغمر الكيميائي و الجدولين (13 و 14) يوضحان النية النتائج التي تم الحصول علية اليها .

الجدول (13) : يوضح قيم الاستقرارية والزحف للإسفلت الاصل والئحور (AS5 ) ومواصفات هيئة الطرق و الجسور العراقية

\begin{tabular}{|c|c|c|c|c|}
\hline MQ & الزحف (MM) & الإستقرارية (KN) & للإسفلت المائة المئوية & الأنموذج \\
\hline 2.21 & 5.1 & 11.2 & 4.75 & $\mathrm{AS}_{0}$ \\
\hline 6.39 & 2.92 & 18.67 & 4.75 & $\mathrm{AS}_{5}$ \\
\hline 3.5 & $2-4$ & 7 & ----- & $\mathrm{AS}_{\text {(S.C.R.B) }}$ \\
\hline
\end{tabular}

الجدول (14) : يوضح قيم الانسلاخ (الانفصال) للإسفلت الاصل و المُحور(AS5 ) 


\begin{tabular}{|c|c|c|c|c|}
\hline 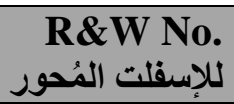 & 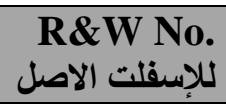 & R\&W No. & نسبة Na2CO3 & الأنموذج \\
\hline ------ & ----- & 1 & 0.025 & ------ \\
\hline ----- & ---- & 2 & 0.041 & ----- \\
\hline ------ & 3 & 3 & 0.082 & $\mathrm{AS}_{0}$ \\
\hline ----- & ----- & 4 & 0.164 & ----- \\
\hline 5 & ----- & 5 & 0.328 & $\mathrm{AS}_{5}$ \\
\hline ----- & ----- & 6 & 0.656 & ----- \\
\hline ----- & ----- & 7 & 1.312 & ----- r r \\
\hline ----- & ----- & 8 & 2.624 & ---- \\
\hline
\end{tabular}

يتضح من كلا الجدولين ان النموذج المحور كان أفضل بكل الدقاييس من الإسفلت الاصل كما ان النموذج المحور كان ذا مواصفات افضل من المو اصفات القياسية المسموح بها.

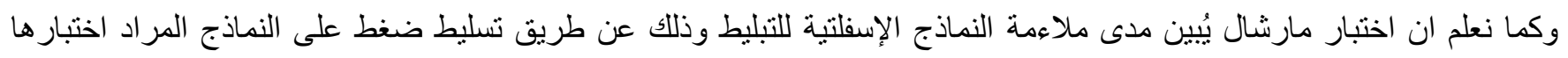

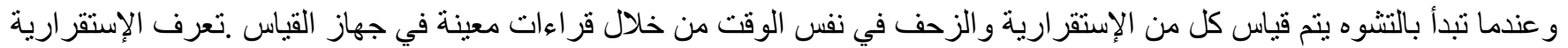

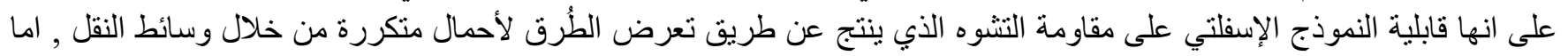

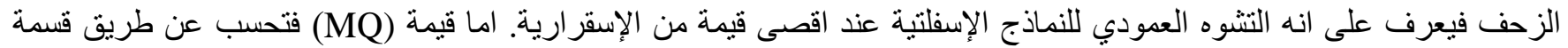

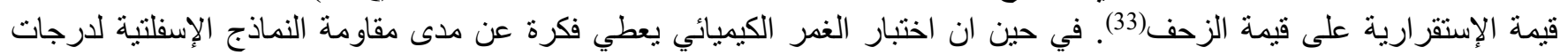

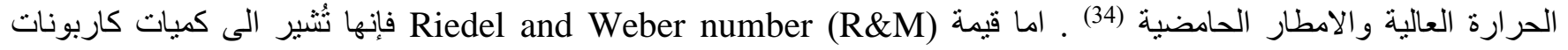
الصوديوم حيث ان الرقم (1) يُشير الى أقل كمية من كاربونات الصوديوم ( 0.025 غم ) , والرقم (8) يُشير الى أعلى كمية من

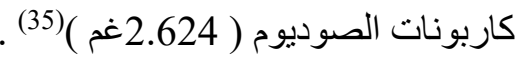

ولغرض معرفة مدى مقاومة النموذج الإسفلتي الئحور والاصلي لعامل الثقادم وتأثره بالعو امل الجوية المختلفة تم دراسة تأثير التقادم

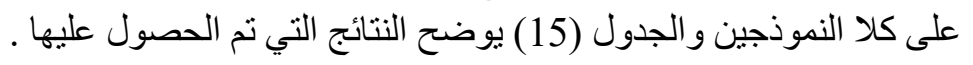

الجدول (15) : الخواص الريولوجية لإسفلت الاصل والإسفلت المُحور بالميلامين قبل وبعد الاخضاع لفحص الفرن للأغثية الرقيقة 


\begin{tabular}{|c|c|c|c|}
\hline بعد فحص الفرن & قِّل فحص الفرن & الخواص الريولوجية & الانموذج \\
\hline $150<$ & $150<$ & $\begin{array}{c}\text { الاسنطالة } \\
\text { (25,cm) }\end{array}$ & \multirow{5}{*}{$\mathbf{A S}_{\mathbf{0}}$} \\
\hline 53 & 50 & درجة الليونة (مه) & \\
\hline 43.5 & 46.7 & 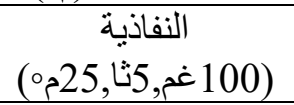 & \\
\hline-0.793 & -1.349 & دليل الاختر اق(PI) & \\
\hline 0.05 & ------ & النسبة المئوية للفقدان & \\
\hline $150<$ & $150<$ & $\begin{array}{c}\text { الاستطالة) } \\
\text { (م)25,cm) }\end{array}$ & \multirow{5}{*}{$\mathbf{A S}_{5}$} \\
\hline 56 & 54.2 & درجة الليونة (مه) & \\
\hline 42.25 & 42.85 & (100 غم, النفاذية 25مة 25م) & \\
\hline-0.199 & -0.555 & دليل الاختر اق(PI) & \\
\hline 0.014 & ------ & النسبة المئوية للفقدان & \\
\hline
\end{tabular}

نلاحظ من الجدول (15) ان درجة تأتر النموذج الإسفلتي المُحور بالتقادم الزمني (زمن ودرجة حرارة) تكون قليلة وضمن الحدود

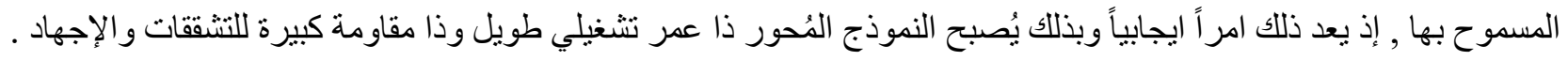

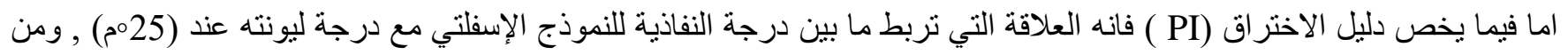

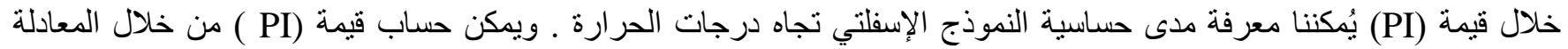
الرياضية النالية (36)

$\frac{20-P I}{10+P I}=50\left(\frac{\log 800-\text { logpent }}{T(R B)-T}\right)$

PI T(RB) T : T Tرة الحرارة التي يتم عندها قياس النفاذية وتساوي (25॰م). Pent

ان النماذج الإسفلتية ذات الموصفات الملائمة للاستخدام في مجال التبليط تمتلك قيم دليل اختر اق (PI) تقع ما بين (+2 و -2) (36) .

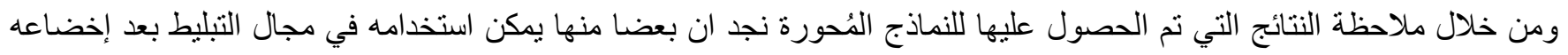

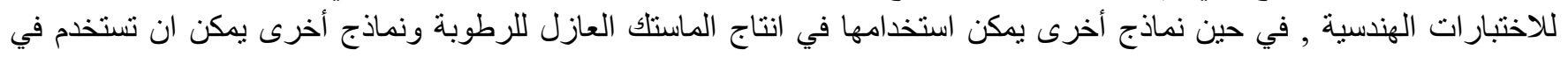

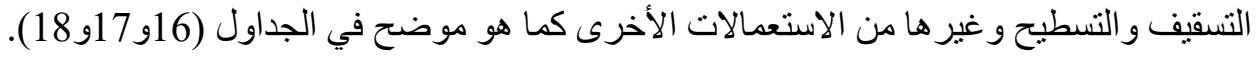

الجدول (16) : المواصفات القياسية للإسفلت المستخلم للتبليط بحسب هيئة الطرق والجسور العراقية [S.C.B.R 38 ]

\begin{tabular}{|c|c|c|}
\hline الحد الأدنى & الحد الأعلى & الخواص الريولوجية \\
\hline 100 & $100<$ & الاستطالة (25,cmم) \\
\hline
\end{tabular}




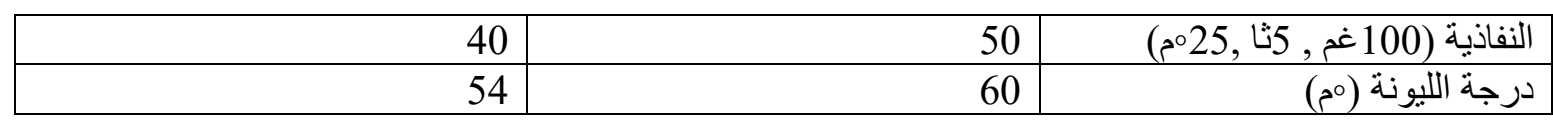

الجدول (17) : المواصفات القياسية العراقية للإسفلت المستعمل في التسطيح( 39 )

\begin{tabular}{|c|c|c|}
\hline الحد الأدنى & الحد الأعلى & الخواص الريولوجية \\
\hline 10 & $10<$ & الاستطالة (25,cm)م \\
\hline 18 & 40 & النفاذية (100 غم, 5ثا ,25॰م) \\
\hline 57 & 66 & درجة الليونة (oم) \\
\hline
\end{tabular}

الجدول (18) : المواصفات القياسية الأمريكية (88 - ASTM(D491 للإسفلت المستعمل في انتاج الماستك (40)

\begin{tabular}{|c|c|c|}
\hline الحد الأدنى & الحد الأعلى & الخواص الريولوجية \\
\hline 15 & $15<$ & الاستطالة (25,cm) \\
\hline 20 & 40 & النفاذية (100 غم , 5ثا ,25॰م) \\
\hline 54 & 65 & درجة الليونة (مم) \\
\hline
\end{tabular}

الاستنتاجات

بعد الانتهاء من هذه الدر اسة يتضح ماياتي :

1- عملية التحوير بالمز ائج البوليمرية كانت عملية ناجحة في مجال الحصول على خواص ريولوجية جيدة .

2 - يعمل كل من الاصق التجاري الذي جُل تكوينه من ال (EVA ) على اعطاء الليونة اللازمة للنموذج المُحور و الميلامين يعطي الصلادة أو الصلابة الازمة كل الاصن التحن

3- يلعب الكبريت دور اً مهماً في عملية التحوير الريولوجي و هو ما اتضح جلياً في هذه الدراسة.

شكر وتقدير

في ختام هذا البحث لا يسعُنا الا ان نتقدم بجزيل الثكر و التقدير الى جامعة الموصل ـ كلية التربية للعلوم الصرفة ـ قسم الكيمياء على بلى

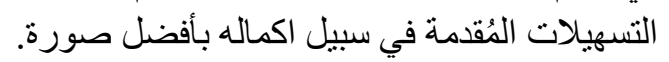

المصادر

1. Al-Dabouni,A.A.,\& Ali,H.,(1986) "Oil of Origin, Installation and Technology ".Mosul University, PP.10-13 ,63-73,65,69-70,166-174,232-236,292-294,298,301,621.

2. Parkash,S., "Petroleum Fuels Manufacturing Hand Book", Mc Graw-Hill Companies, (2010), Inc. 102-131.

3. "Introduction to asphalt",(2001), Asphlat Institute Manual Saries No.(5),(MS-5),p.2,9$11,14,61$.

4. Zhang, L. and Green field "Effects of Polymer Modifiction on Properties and Microstructure of Model asphalt Systems”, Energy \& Fuels, Vol. 22, ,(2008) pp. 3363-3375 . 
5. Ramadan,A.M., Al-Ghanam,Sh.A., and DhanounA.A.,(1991) "Industrial Chemistry and Industrial Pollution", The Book House for Printing and Publishing,University of Mosul PP.119,421,465-469,523-524.

6. Hubson, G.D.,(1973), “Modern Petrolem Technologe”, Vol.4 ${ }^{\text {th }}$ ed., Ltd. Britain, pp. 804-806.

7. Schmerling, L.,(1982). "Encyclopedia of Science and Technology".Vol.10,5" ed., Mc GrwHill, Inc. New York ,pp-76.

8. Lesueur D., (2009), "the Colloidal Structure of Bitumen, Consequences on the Rheology and on the Mechanesms of Modification" , Advances in Colloid and Interrface Science, pp. 145, 28-42.

9. Cunha,T.N.F., Cardoso,G., and Frota,C.A.,(2017) "Rheological Properties of Asphalt Modified With Natural Fibers and Oxidants".Journal of Multidisciplinary Science and Technology, Vol .4Issue10,ISSN:2458-9403.

10. Adil, A.,(2018) "Utilization of Waste Plastic Water Bottle as a Modifier For Asphalt Mixture Properties". Journal of Engineering and Development, Vol.20,No.2,ISSN: 1813-7822

11. Gao,C., Wu,W., (2018) "Using ESEM to Analyze the Microscopic Property of Basalt Fiber Reinforced Asphalt Concrete". International Journal of Pavement and Technology 374-380(In Chinese).

12. Peres, I.P.,Pasandin, A.M.R., and Pereira, P.A.A.,(2019) "Use of Lignin Biopolymer from Industrial Waste as Bitumen Extender for Asphalt Mixtures". Journal of Cleaner.

13. Fournier,M.F.,Alvarez,d.a. and Tenza-Abril,A.G.,(2020) "Combining Reclaimed Asphalt Pavement (RAP) and Recycled Concrete Aggregate (RCA)from Cuba to Obtain a Coarse Aggregate

14. Ahmed,S.S.,Hamdoon,A.A.,(2020) "The Use of Lignin in the Rheological Modification of Dura Asphalt" Journal of Education and Science, Vol.29,No.4,pp.22-41,ISSN 1812-125X.

15. Ahmed,S.S.,Hamdoon,A.A.,(2020) "The Rheological Properties of Asphalt by Commercial Adhesive (Ethylene-Vinyl Acetate) and Air Blowing Process" Journal of Education and Science, Vol.29,No.3,pp.102-118,ISSN 1812-125X.

16. Joohari,I.B.,Giustozzi,F.,(2021) "Effect of Different Vinyl-Acetate Contents in Hybrid SBSEVA modified Bitumen"Civil and Infrastructure Engineering, Royal of Technology (RMIT)University,376-392 Swanston St.Melbourne, VIC3000,Australia.

17. Mashaan,M.,Nikraz,H.,(2021) "Investigating the Engineering Properties of Asphalt Binder Modified with Waste Plastic Polymer"Article in Shams Engineering Journal ;DOI:101016/J.asej.035.

18. Pakenari,M.M.,Hamedi,H.,(2021) "Investigating the Effective Laboratory Parameters on the Stiffness Modulus and Fatigue Cracking of Warm Mix Asphalt" Article in International Journal of civil Engineering ; DOI:10.1007/S40999-020-00592-7.

19. Hamedi,H.,Joubani,A.,S.,(2021) "AnExperimental Investigation into the Effect of Asphalt Binder Modified with SBR Polymer on the Moisture Susceptibility of Asphalt Mixtures" Article in Periodica Polytechnica Civil Engineering;DOI:10.3311/PPci.16691. 
20. Reyes-Labarta,J.A.,\&Marcilla,A.,(2012). “Thermal Treatment and Degradation of Crosslinked Ethylene Vinyl Acetate Polyethylene-Azodicardonamide $\mathrm{ZnO}$ Foams. Complete Kinetic Modelling and Analysis" Industrial \& Engineering Chemistry Research.51(28):9515-9530.

21. American Society for Testing and Materials, (D113-07),(2008).

22. American Society for Testing and Materials, (D36-95),(2000).

23. American Society for Testing and Materials, (D5/D5M-13),(2013).

24. Ali , L. H. , Al-Ghannam, K. A "Investigation in to Asphaltenes in Heavy Crude Oils" ,J.Fuel, Vol. 60, (1981) pp: 1043-1046.

25. American Society for Testing and Materials, (D1559-2004).Standard Specification, section 4,Vol., 04-03.

26. American Society for Testing and Materials, D1754-97R,Section 4, Vol . 04. 03, ,(2002) Road and Paving Materials Vehicle-Pavement Systems, New York, Ny.

27. Speight,T.G ,2015) “Asphalt Materials Science and Technology” Butterworth-Heinemann., pp. 232-233.

28. ASTM D1754-97R "Standard test method for effect of heat and air on asphaltic materials (Thin Film Oven Test)",Section 4,Vol .04 .03, (2002), Road and paving materials-vehiclepavement systems, New york, Ny.

29. Dekhli S., Mokhtar K.A., and Bachir D.S., (2015) "Rheological Behaviour of Ethylene-Vinyl acetate (EVA) Modifi Road Bitumen”. Journal of Applied Sciences Vol. 15, No.(3),PP444455.

30. Abd-Alaziz,A.N., (2004) "Study The Rroperties of Asphalt Modifeid By Polymers" Master Thesis University of Mosul College of Education for Pure Science.

31. Tang, N., Huang,W., Hu,J., and Xiao, F.,(2018) "Rheological Characterisation of Terminal Blend Rubberised Asphalt Binder Contaning Polymeric additive and Sulphur" . Road Materials and Pavement Design,19(6),1288-1300.

32. Kuriacose J. C., Rajarm J.,(1984), "Chemistry in Engineering and Technology". Vol.2, Tata McGraw-Hill Publishing Company Limited, New Delhi .

33. Zoorob, S.E. and Suparma, L.B., (2000), "Laboratory Design : an Investigation of the Properties of Continuously Graded Asphalt Concrete Containing Recycled Plastic Aggregate Replacement (Plastiphalt)", Chem Cencr Compos ; 22:233-242.

34. Chemical Immersion Standard method TMH1 (Road Research Laboratory1986), England / Static Immersion ASTM (D1664).

35. Speight J.G.,(2015), “Asphalt Materials Science and Technology" ButterworthHeinemann.PP.232-233.

36. Al-Frekh and Abu Shihada A., (1981), "Production of Bitumen Grades", Brit, UK Pat. Appl GB2,280, 406, Chem. Abs.(1982), Vol.96,126085g.

37. Hubson, G.D.,(1975), "Modern Petrolem Technologe”, Ltde Britaen , 4th ed., PP.810,811.

38. State Commission for Roads and Bridges (SCRB)(2001),Department of Design and Study , Ministry of Construction and Housing and Municipalities and Public Works, Baghdad.

39.Standard Specfication No.1196 of 1988 Issued by the Central Agency for Measurement and Quality Control of the Bitumen Used for Flatness.

40. ASTM, Part 11, (D491-41), (1969),P. 250-251. 